Europhys. Lett., 54 (3), pp. 329-334 (2001)

\title{
The settling of fine cohesive powders
}

\author{
J. M. Valverde ${ }^{1}$, M. A. S. Quintanilla ${ }^{1}$, A. Castellanos $^{1}$ and P. Mills ${ }^{2}$ \\ 1 Departamento de Electrónica y Electromagnetismo, Facultad de Física \\ Avda. Reina Mercedes s/n, 41012 Sevilla, Spain \\ 2 Laboratoire de Physique des Matériaux Divisés et des Interfaces \\ Université de Marne-la-Vallée, Bâtiment Lavoisier \\ 5 Bvd. Descartes, F-77454 Marne-la-Vallée Cedex 2, France
}

(received 5 December 2000; accepted in final form 19 February 2001)

PACS. 47.55.Kf - Multiphase and particle-laden flows.

PACS. 47.55.Mh - Flows through porous media.

PACS. 61.43.Gt - Powders, porous materials.

\begin{abstract}
We investigate the settling of an initially fluidized bed of a fine cohesive powder. Experimental results on the sedimentation rate cannot be fitted by theoretical and empirical equations existing in the literature and derived for non-cohesive spheres. We extend these equations to account for the adhesion-induced aggregation of fine particles. The number of particles per aggregate $N$ and the ratio of aggregate radius $R$ to particle radius, $a$, have been chosen as fitting parameters. Results from two independent approaches are in very good agreement with the experimental data for approximately the same values of $N$ and $R$. Pictures taken from the fluidized bed show that the average aggregate radius matches the predicted one.
\end{abstract}

Introduction. - The fluidization of fine powders is of fundamental importance to many industries. The aim of this paper is to consider the sedimentation rate of homogeneously fluidized fine particles after gas supply to an initially fluidized bed is stopped, and to relate it with the microstructure of the fluidized bed. The relationship between the superficial fluidizing velocity (or the settling velocity in sedimentation) and the solid volume fraction for a homogeneous fluidized powder of non-cohesive spheres was well described by the phenomenological Richardson-Zaki law [1]

$$
\frac{v_{\mathrm{s}}}{v_{\mathrm{p} 0}}=(1-\phi)^{n}
$$

Here $\phi$ is the solid volume fraction and $v_{\mathrm{p} 0}$ is the sedimentation velocity of a single particle, $v_{\mathrm{p} 0}=(2 / 9)\left(\rho_{\mathrm{p}}-\rho_{\mathrm{f}}\right) a^{2} g / \mu$ in the laminar regime, where $\rho_{\mathrm{p}}$ is the particle density, $\rho_{\mathrm{f}}$ is the gas density, $g$ is the gravity field, $a$ is the particle radius, $\mu$ is the viscosity of the gas and $n$ a parameter of order 5 in the small Reynolds number limit. Theoretical considerations by Batchelor [2] leaded to $v_{\mathrm{s}} / v_{\mathrm{p} 0} \simeq 1-5.6 \phi$ for very diluted suspensions of non-Brownian hard spheres in the limit of small Reynolds numbers. Thus, the sedimentation velocity predicted by eq. (1) conforms with the dilute limit by Batchelor for $n=5.6$. Mills and Snabre [3] proposed a simple model for the settling velocity of a system of concentrated non-cohesive monodisperse (c) EDP Sciences 
spheres based on the energy balance during the sedimentation process. They used mean-field and scaling arguments to arrive at the expression:

$$
\frac{v_{\mathrm{s}}}{v_{\mathrm{p} 0}}=\frac{1-\phi}{1+k \phi /(1-\phi)^{3}} .
$$

They chose the parameter $k=4.6$ to match eq. (2) in the diluted limit $\left(v_{\mathrm{s}} / v_{\mathrm{p} 0}=1-(k+1) \phi\right)$ to Batchelor equation and reported an excellent agreement with experimental data. The agglomeration of dry fine particles in fluidized beds, due to the attractive van der Waals force, is a well-known phenomenon [4]. Recently, Lettieri et al. [5] reported on deviations from the Richardson-Zaki law in the settling of FCC catalysts powders (average particle size $50 \mu \mathrm{m}$ ) when contact forces between particles were increased by the presence of potassium acetate. For our experimental fine powders (particle size $\sim 10 \mu \mathrm{m}$ ) the van der Waals interparticle adhesive force exceeds by several orders of magnitude the particle weight [6]. For this reason these fine particles are expected to be aggregated in the fluidized regime. In this paper we show that settling experiments on these powders confirm this expectation. We propose an extension of the existing models for the sedimentation of non-cohesive grains to account for the cohesivity of fine particles.

Experimental work. - In our experiments we have used a commercially available xerographic toner (Canon CLC700). Particle density $\left(\rho_{\mathrm{p}}=1199 \mathrm{~kg} / \mathrm{m}^{3}\right)$ was measured by using an AccuPyc 1330 Pycnometer. Toner particles are based on a random copolymer, their volume average diameter is $8.53 \mu \mathrm{m}$, they are rather monodisperse (standard deviation from the average diameter $2.32 \mu \mathrm{m}$ ) and irregularly shaped. Even though particle size would lead one to predict a type-C behavior (impossible to fluidize) in the Geldart classification scheme [7], the addition of flow conditioners (silica nanoparticles, which are dispersed on the powder particle surfaces) causes a shift to type-A behavior i.e. this powder can be driven into a homogeneous fluidization phase [6]. The powder sample is held in a $4.45 \mathrm{~cm}$ diameter, $17 \mathrm{~cm}$ height cylinder made of polycarbonate, the base of which is a sintered metal filter of $5 \mu \mathrm{m}$ pore size, and of width $1.5 \mathrm{~mm}$. A dry nitrogen (dynamic viscosity $\mu=1.89 \times 10^{-5} \mathrm{Pas}$ at ambient temperature) tank furnished with a mass flow controller supplies adjustable gas flow. By using dry nitrogen, the effects of moisture on the interparticle adhesion are minimized. Charge spectrograph measurements made at Xerox Co. indicate that electrostatic forces are minimal. All the measurements start by driving the powder into the freely bubbling phase. Once the powder is in the bubbling phase, the fluidizing gas is set down to a given value below the minimum bubbling point and the powder settles to a reproducible and stable fluidized state. The bed height is read from an ultrasonic sensor and from this data the powder solid volume fraction is calculated. In fig. 1 the superficial gas velocity is represented against the fluidized powder solid volume fraction. For superficial gas velocities above $0.77 \mathrm{~cm} / \mathrm{s}$ (minimum bubbling point) the mean bed height begins to decrease slowly, with marked oscillations of the free surface as the gas bubbles burst. Once the powder reaches a steady state for a given fluidization velocity, the gas flow is abruptly reduced to zero and the powder settles down. During the sedimentation process the bed height is continuously monitored by the ultrasonic sensor. In fig. 2 we represent a typical sedimentation curve. From this figure it is clear that the head of the bed falls initially at a constant velocity, but as the bed approaches the settled state it slows down, indicating the formation of stresses carried by interparticle contacts that hinder sedimentation [8]. We have measured, for different initial superficial velocities of the fluidizing gas, the initial settling velocity of the head of the bed. Results are shown in fig. 3 . From the frame where the gas is at rest the particles in the fluidized bed are settling at a velocity equal to the superficial gas velocity. Thus, as we would expect, the settling velocity 


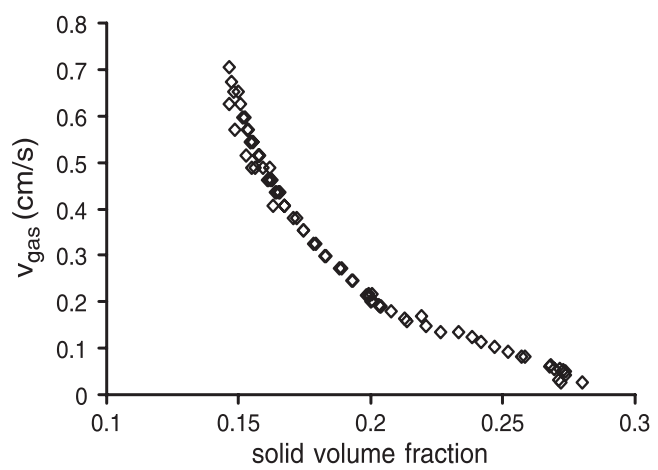

Fig. 1

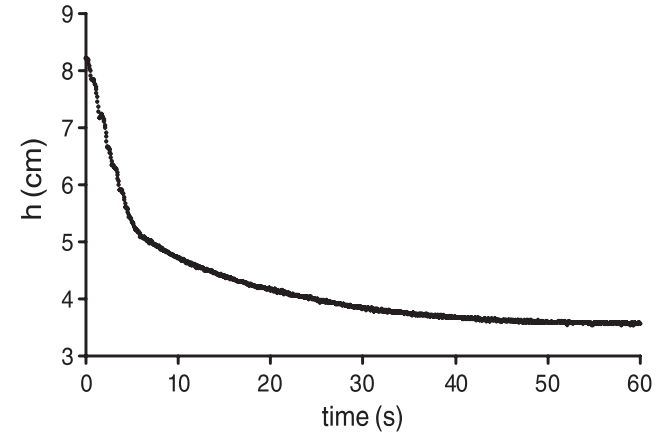

Fig. 2

Fig. 1 - Superficial gas velocity as a function of the solid volume fraction of the fluidized powder.

Fig. 2 - Powder bed height as a function of time after the gas supply has been suddenly stopped for an initial superficial gas velocity of $0.54 \mathrm{~cm} / \mathrm{s}$.

immediately after the gas is stopped is, within the experimental scatter, equal to the superficial gas velocity in the homogeneous fluidization phase. If the gas velocity passes the minimum bubbling velocity $(\simeq 0.77 \mathrm{~cm} / \mathrm{s})$ the initial settling velocity decreases slightly because in the bubbling regime part of the gas rises in the form of bubbles. In fig. 4 the settling velocity is represented against the initial solid volume fraction of the fluidized powder for gas velocities below the minimum bubbling velocity. As can be seen from this figure, the Richardson-Zaki (eq. (1)) and Mills-Snabre (eq. (2)) predicted results are in agreement, but both laws clearly underestimate the experimental results. We conclude from this that the particles do not behave as individuals in the fluidized bed but are aggregated. Otherwise, the experimental data can be very well fitted by the equation $v_{\mathrm{s}} / v_{\mathrm{p} 0}=\alpha(1-\beta \phi)^{5.6}$, with $\alpha=16.76$ and $\beta=1.947$. In the next section we will analyze the physical meaning of this fit.

Theoretical analysis. - From our experimental work it is clear that eqs. (1) and (2) are not suitable for aggregated systems. The simplest approximation to account for this effect is to assume their validity for the settling of a system of monodisperse aggregates. Then the Richardson-Zaki law would be

$$
\frac{v_{\mathrm{s}}}{v_{\mathrm{a}}}=\left(1-\phi_{\mathrm{ef}}\right)^{n},
$$

where $v_{\mathrm{a}}$ is the settling velocity of an individual aggregate and $\phi_{\text {ef }}$ is the volume fraction occupied by the aggregates. The settling velocity of an individual aggregate can be calculated from the balance of the weight of the aggregate, $F_{\mathrm{G}}=N(4 / 3) \pi a^{3} \rho_{\mathrm{p}} g$, and the Stokes force, $F_{\mathrm{S}}=6 \pi \eta R v_{\mathrm{a}}$, where $N$ is the number of particles aggregated and $R$ the hydrodynamic radius of the aggregate. Note that the buoyancy force is neglected since the density of the gas is much smaller than the density of the particle. From this we have $v_{\mathrm{a}}=v_{\mathrm{p} 0} N / \kappa$, where $\kappa \equiv R / a$. The volume fraction of the aggregates $\phi_{\text {ef }}$ would be given by $\phi_{\text {ef }}=\left(N_{0} / N\right)(4 / 3) \pi R^{3}$, where $N_{0}$ is the number density of particles $\left(\phi=N_{0}(4 / 3) \pi a^{3}\right)$ and we have made the important assumption that the geometric radius of the aggregate can be approximated by its hydrodynamic radius. Thus $\phi_{\text {ef }}=\phi \kappa^{3} / N$ and eq. (3) would turn into

$$
\frac{v_{\mathrm{s}}}{v_{\mathrm{p} 0}}=\frac{N}{\kappa}\left(1-\phi \frac{\kappa^{3}}{N}\right)^{n},
$$




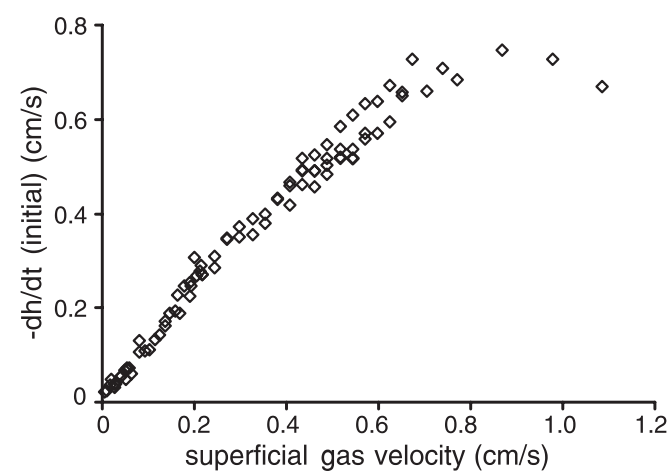

Fig. 3

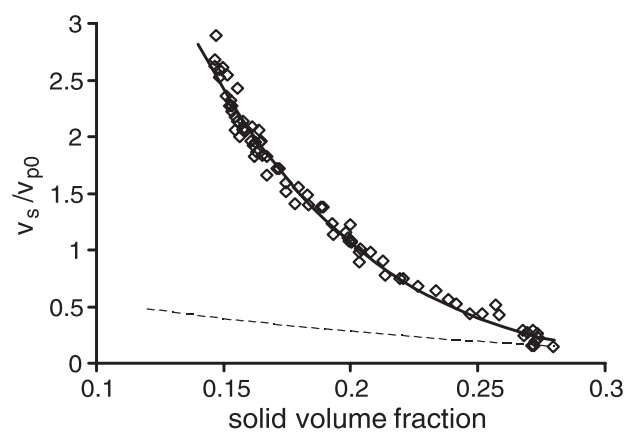

Fig. 4

Fig. 3 - Initial settling velocity of the head of the powder bed as a function of the superficial gas velocity to which the powder was previously fluidized.

Fig. 4 - Initial settling velocity of the head of the powder bed (normalized by the settling velocity of an isolated particle) as a function of the solid volume fraction of the powder just before the gas supply was stopped. Experimental data, predicted results (- - ) from eq. (1) and eq. (2) and best-fit curves (-) from eq. (4) for $N=96, R / a=5.712$ and eq. (7) for $N=124, R / a=6.577$ are shown.

which is in the form of the best-fit equation to the experimental data. Thus in this simple model $\alpha=N / \kappa$ and $\beta=\kappa^{3} / N$, giving for the best fit $N=96$ and $\kappa=5.712$. The aggregate diameter is then $2 R=2 a \kappa \simeq 97 \mu \mathrm{m}$. To check for the existence of particle aggregates, we have visualized the structure of the fluidized bed. Powder particles have a great tendency to adhere to a paper surface. We made use of this and a paper card was carefully lowered edgewise into the fluidized bed so as to cleave it perpendicular to the gas flow path. Then, on carefully withdrawing the card, it was found to carry a replica of the fluidized powder. This technique introduces an unavoidable shear on the sample but we expect it to be small due to the low values of the extracting velocity. Figure 5 shows a picture from the optical microscope. Particle aggregates of size of the order of $100 \mu \mathrm{m}$ can be distinguished. Moreover, as we assumed in the model, the aggregate size does not change appreciably with the solid
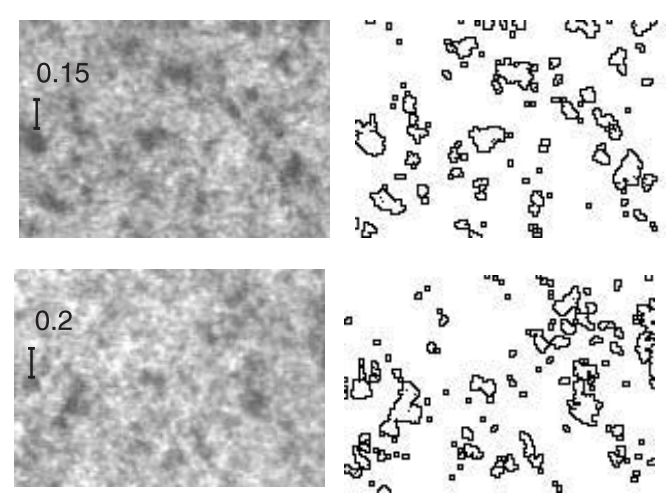

Fig. 5 - Pictures of the aggregates corresponding to different solid volume fractions (size of the bar $150 \mu \mathrm{m})$. The contour of the aggregates has been outlined by an image software. 
volume fraction. According to our model, the packing fraction of the particles in the aggregate would be given by $\phi_{*}=N / \kappa^{3}=0.514$, which is below the random isostatic packing limit for hard spheres $\left(\phi_{*}=0.57[3]\right)$. As we would expect, this value is larger than the average solid volume fraction of the settled powder $(\phi \simeq 0.36)$. Aggregates settle in open structures due to the strong cohesive forces that oppose gravity and restructuring and lead to small average values of the solid volume fraction. When the bed fluidizes the weaker interparticle contacts are broken, leaving free compact agglomerates of strongly attached particles that cannot be further disrupted. This interpretation would also explain the independence of the aggregate size from the range of solid volume fractions of our fluidized beds.

Let us now turn to a new derivation of eq. (2) in our model of aggregates settling. The variation rate of the potential energy per unit volume of the bulk suspension is given by [3]

$$
d_{\mathrm{t}} W_{\mathrm{g}}=-\phi(1-\phi) \rho_{\mathrm{p}} g v_{\mathrm{s}},
$$

where we have assumed $\rho_{\mathrm{p}} \gg \rho_{\mathrm{f}}$. On the other hand, we would assume that the dissipated energy per unit time and per unit volume in the system of aggregates can be approximated by $[3]$

$$
d_{\mathrm{t}} D=(9 / 2) \frac{\phi_{\mathrm{ef}} \mu v_{\mathrm{s}}^{2}}{R^{2}}\left(1+k \phi_{\mathrm{ef}} /\left(1-\phi_{\mathrm{ef}}\right)^{3}\right),
$$

i.e. from the point of view of the dissipated energy the aggregates behave as particles with an effective radius $R=\kappa a$ and a volume fraction $\phi_{\mathrm{ef}}=\left(\kappa^{3} / N\right) \phi$. The dissipated energy must balance the loss of potential energy i.e. $d_{\mathrm{t}}\left(W_{\mathrm{g}}+D\right)=0$ and from this balance we have

$$
\frac{v_{\mathrm{s}}}{v_{\mathrm{a}}}=\frac{1-\phi}{1+k \phi_{\mathrm{ef}} /\left(1-\phi_{\mathrm{ef}}\right)^{3}}
$$

where $v_{\mathrm{a}}$ and $\phi_{\text {ef }}$ are related to aggregate properties as before. If we now fit eq. (7) to the experimental data we obtain $N=124$ and $\kappa=6.577$ (see fig. 4). Note that these values are quite close to the ones obtained from the modified Richardson-Zaki empirical law. Furthermore, the best-fit curves from eq. (4) and eq. (7) to the experimental data are almost identical in our range of solid volume fractions.

Conclusion. - Fine powders settle at larger velocities than the predicted ones from empirical and theoretical models for non-cohesive spheres. This disagreement can be explained by the formation of aggregates due to interparticle adhesive forces which exceed by several orders of magnitude the particle weight. A straightforward extension of the RichardsonZaki [1] empirical model and the theoretical Mills-Snabre [3] model, originally developed for the settling of non-cohesive spheres, allows us to account for the settling of aggregates characterized by a number of particles per aggregate $N$ and an effective radius $R$. Both models give results which are in very good agreement with the experimental data for approximately the same values of $N$ and $R$. From this study we can conclude that the average number of particles per aggregate in our homogeneous fluidized powder is $N \simeq 100$, and that the average aggregate radius is $R \simeq 6 a \simeq 50 \mu \mathrm{m}$ independent of the solid volume fraction. Furthermore, the particles in the aggregate are more closely packed than the average in the settled powder. These results have been corroborated by direct visualization of samples taken from the fluidized bed. We have found that the settling properties described in this paper for a particular powder are extensible to other fine cohesive powders. We will report on these measurements on other fine cohesive powders elsewhere. 
This research has been supported by the Xerox Foundation, by the Spanish Government Agency Dirección General de Ciencia y Tecnología (DGES) under contract PB96-1375 and by NATO Grant LINKAGE PST.CLG.976575.

\section{REFERENCES}

[1] Richardson J. F. and Zaki W. N., Trans. Inst. Chem. Eng., 32 (1954) 35.

[2] Batchelor G. K., J. Fluid Mech., 119 (1982) 379; Batchelor G. K. and Wen C.-S., J. Fluid Mech., 124 (1982) 495.

[3] Mills P. and Snabre P., Europhys. Lett., 25 (1994) 651.

[4] Zhou T. and Hongzhong L., Powder Technol., 101 (1999) 57.

[5] Lettieri P., Yates J. G. and Newton D., Powder Technol., 110 (2000) 117.

[6] Castellanos A., Valverde J. M., Perez A. T., Ramos A. and Watson P. K., Phys. Rev. Lett., 82 (1999) 1156.

[7] Geldart D., in Gas Fluidization Technology, edited by D. Geldart (Wiley, New York) 1986.

[8] Valverde J. M., Castellanos A. and Quintanilla M. A. S., Phys. Rev. Lett., 86 (2001) 3020. 